\title{
An investigation of the molecular mechanism, chemoselectivity and regioselectivity of cycloaddition reaction between acetonitrile N-Oxide and 2,5-dimethyl-2H-[1,2,3]diazaphosphole: a MEDT study
}

\author{
ABDELLAH ZEROUAL ${ }^{\mathrm{a}, *}$, MAR RÍOS-GUTIÉRREZ ${ }^{\mathrm{b}}$, MOHAMMED SALAH $^{\mathrm{a}}$, \\ HABIB EL ALAOUI EL ABDALLAOUI ${ }^{\mathrm{a}}$ and LUIS RAMON DOMINGO ${ }^{\mathrm{b}}$ \\ ${ }^{a}$ Molecular Modeling and Spectroscopy Research Team, Faculty of Science, Chouaib Doukkali University, \\ P.O. Box 20, 24000 El Jadida, Morocco \\ ${ }^{b}$ Department of Organic Chemistry, University of Valencia, Dr. Moliner 50, 46100 Burjassot, Valencia, \\ Spain \\ E-mail: zeroualabdellah2@gmail.com
}

MS received 14 May 2019; revised 16 June 2019; accepted 23 June 2019

\begin{abstract}
The [3+2] cycloaddition (32CA) reactions of acetonitrile N-oxide with 2,5-dimethyl-2H$[1,2,3]$ diazaphosphole has been studied using the Molecular Electron Density Theory (MEDT) through DFT calculations at the B3LYP/6-31G(d,p) computational level. Analysis of the relative free energies associated with the competitive ortho and meta reaction paths shows high chemo- and regioselectivity for this 32CA reaction in clear agreement with the experimental outcomes. The topological analysis of the electron localization function (ELF) of the selected points of the IRC associated with the formation of the P-C and $\mathrm{C}-\mathrm{O}$ single bonds indicates a zwitterionic type structure. The 32CA reaction takes place through a two-stage one-step mechanism initialized with the formation of the P-C single bond.
\end{abstract}

Keywords. Diazaphosphole; acetonitrile N-oxide; MEDT; chemoselectivity; regioselectivity.

\section{Introduction}

The studies regarding the preparation of isoxazoline products were the object of extensive investigations in the recent years. ${ }^{1,2}$ This is due to their amazing chemical properties that make these very useful in many fields like veterinary medicine, chemical synthesis, etc. In veterinary medicine, these are used as highly effective drugs on ectoparasites. ${ }^{3}$ In chemical synthesis, these serve as intermediates in short, stereoselective syntheses of diverse natural and unnatural products. ${ }^{4}$ A variety of synthetic methods have been developed for the preparation of isoxazolines of which the most convenient and attractive route is probably the $[3+2]$ cycloaddition (32CA) reaction of nitrile oxides to alkenes. ${ }^{5}$ In the context of such reactions, we have focused our work on the reaction between 2,5dimethyl-2H-[1,2,3]diazaphosphole $\mathbf{1}$ and acetonitrile $\mathrm{N}$-oxide 2, which leads chemo- and regioselectively to

*For correspondence a single product $\underline{\mathbf{3}}$ (Scheme 1) realized by the group of Yeung Lam. ${ }^{6}$

The current progress made in the theoretical comprehending of 32CA reactions based on the Molecular Electron Density Theory ${ }^{7}$ (MEDT) has allowed creating a very good correlation among the electronic structure of three-atom-components (TACs) and their reactivity. ${ }^{8}$ Therefore, depending on the electronic structure of the simplest TACs, 32CA reactions have been categorized into pdr-type, pmr-type, zw-type and cb-type reactions, ${ }^{8}$ in such a method that while $p d r$-type 32CA reactions can be approved out easily, zw-type 32CA reactions demand adequate nucleophilic/electrophilic activations to take place ${ }^{9}$ (Chart 1 ).

A recent MEDT investigation of the reactivity and regioselectivity of the 32CA reaction between benzonitrile oxide $\underline{\mathbf{a}}$ with methyl acrylate $\underline{\mathbf{b}}$ (Scheme 2) explain that this zwitterionic TAC has a low nucleophilic and electrophilic character. This makes the reaction a non-polar process and, hence, the activation energies that are principally associated to the rupture

Electronic supplementary material: The online version of this article (https://doi.org/10.1007/s12039-019-1656-z) contains supplementary material, which is available to authorized users. 


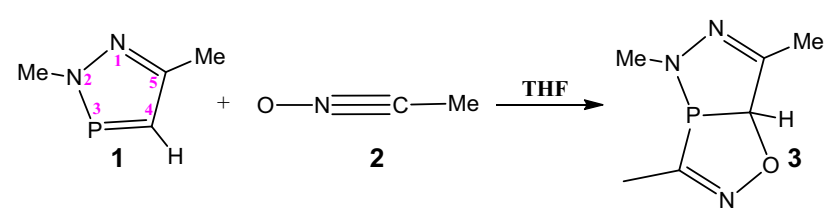

Scheme 1. 32CA reaction of 2,5-dimethyl-2H-[1,2,3]diazaphosphole $\mathbf{1}$ with acetonitrile $\mathrm{N}$-oxide $\mathbf{2}$ in presence of THF as a solvent.

of the C-N triple bond of nitrile oxide, are somewhat high, $11.4 \mathrm{kcal} \cdot \mathrm{mol}^{-1.10}$ This zw-type $32 \mathrm{CA}$ reaction demonstrated poor ortho regioselectivity given that the transition state (TS) associated with the formation of meta isoxazoline d was established to be only 1.6 $\mathrm{kcal} \cdot \mathrm{mol}^{-1}$ less favorable than that associated with the formation of ortho isoxazoline c.

Herein, an MEDT study of the 32CA reaction between acetonitrile N-oxide 2 and 2,5-dimethyl-2H$[1,2,3]$ diazaphosphole $\mathbf{1}$, is performed in order to unveil the molecular mechanism that manage the chemo- and regioselectivity of this reaction.

\section{Computational methods}

DFT computations were executed using the B3LYP functional ${ }^{11,12}$ together with the $6-31 \mathrm{G}(\mathrm{d}, \mathrm{p})$ basis set. ${ }^{13}$ Optimisations were carried out utilizing the Berny analytical gradient optimisation method. ${ }^{14,15}$ The stationary points were characterised by frequency calculations in order to prove that TSs have one and only one imaginary frequency. The intrinsic reaction coordinate ${ }^{16}$ (IRC) paths were drawn in order to verify the energy profiles relating each TS to the two associated minima of the proposed mechanism employing the second-order González-Schlegel integration method. ${ }^{17,18}$ Solvent effects of tetrahydrofuran (THF) were taken into account by full optimization of the gas phase structures using the polarisable continuum model (PCM)
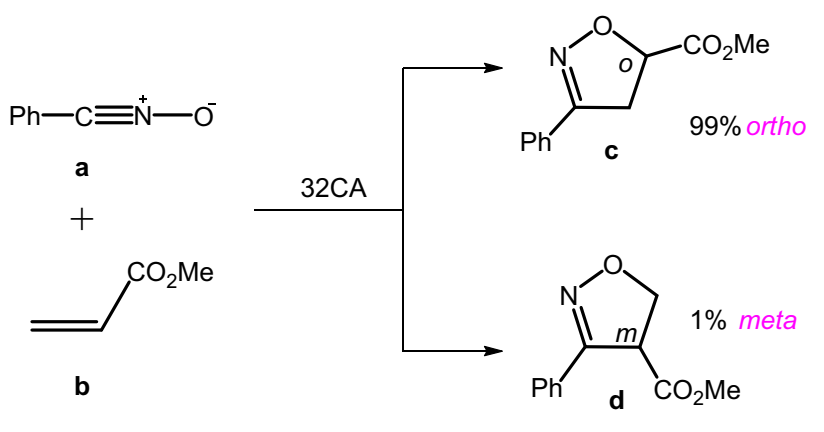

Scheme 2. 32CA reaction of benzonitrile oxide $\underline{\mathbf{a}}$ with methyl acrylate $\underline{\mathbf{b}}$.

developed by Tomasi's group ${ }^{19}$ in the framework of the self-consistent reaction field ${ }^{20-22}$ (SCRF). Values of enthalpies, entropies and Gibbs free energies in THF were calculated with standard statistical thermodynamics at $273.15 \mathrm{~K}$ and $1 \mathrm{~atm}$. The global electron density transfer ${ }^{23}$ (GEDT) was calculated by the sum of the natural atomic charges (q), obtained by a Natural Population Analysis, ${ }^{24,25}$ (NPA), of the atoms belonging to each framework (f) at the TSs; i.e., GEDT (f) $=\sum_{q \in f} q$. The sign shows the direction of the electron density flux in such a manner that positive values mean a flux from the considered framework to the other one.

The global electrophilicity index ${ }^{26} \omega$ was given by the following expression, in terms of the electronic chemical potential $\mu$ and the chemical hardness $\eta$. Both quantities could be approached in terms of the one-electron energies of the frontier molecular orbital HOMO and LUMO, $\varepsilon_{H}$ and $\varepsilon_{L}$ as $\mu=\frac{\varepsilon_{H}+\varepsilon_{L}}{2}$ and $\eta=\varepsilon_{L}-\varepsilon_{H}$, respectively. The empirical nucleophilicity index $\mathrm{N}^{27}$ based on the HOMO energies obtained within the Kohn-Sham and defined as $\mathrm{N}=$ $\mathrm{E}_{\mathrm{HOMO}}(\mathrm{Nu})-\mathrm{E}_{\text {Номо }}(\mathrm{TCE})$ the nucleophilicity was referred to as tetracyanoethylene (TCE). Electrophylic and nucleophilic Parr functions were obtained through the analysis of the Mulliken atomic spin density (ASD) of the

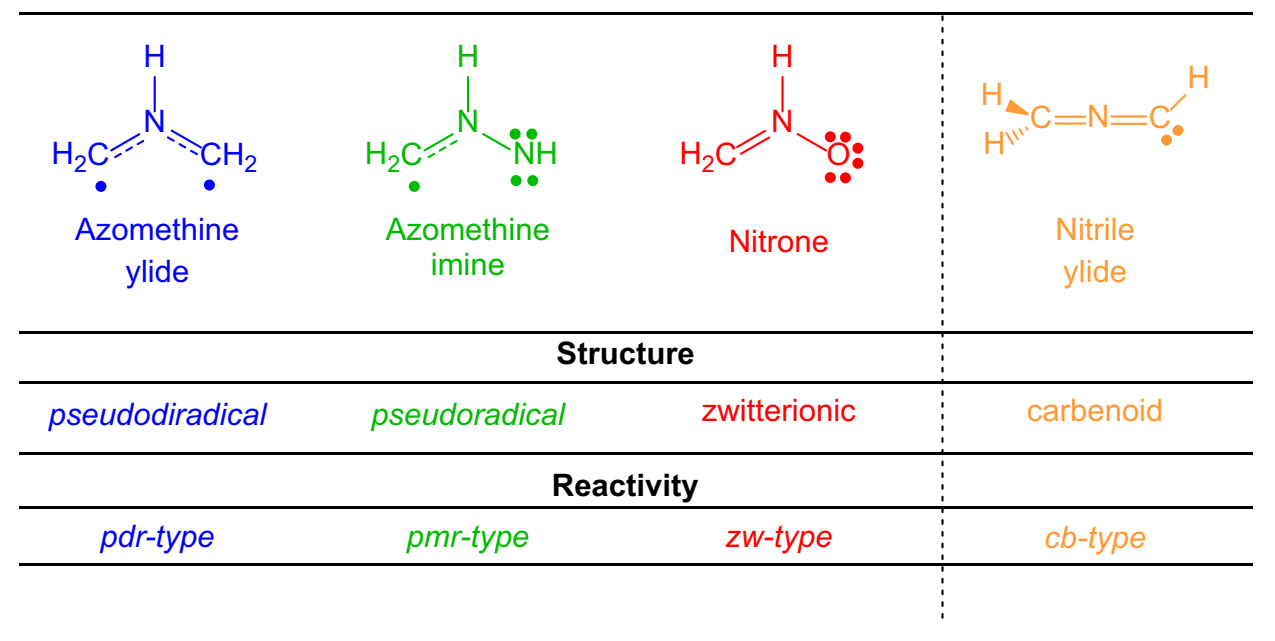

Chart 1. Electronic structure of TACs and the proposed reactivity types in 32CA reactions 
radical anion and radical cation of the reagents. ${ }^{28}$ All computations were carried out with the Gaussian 09 suite of programs. $^{29}$

Topological analyses of the Electron Localisation Func$\operatorname{tion}^{30}$ (ELF) were performed with the TopMod ${ }^{31}$ package using the corresponding mono determinantal wave functions. The characterization of the bond formation processes along the most favourable reaction path was carried out by performing the topological analysis of the ELF for some selected structures along with the IRC profile.

\section{Results and Discussion}

The present MEDT study has been divided into four sections: (i) first, the CDFT reactivity indices of the reagents are analysed; (ii) in the second part, ELF analysis of the electronic structure of acetonitrile $\mathrm{N}$-oxide $\mathbf{2}$ is performed; (iii) the competitive reaction paths associated with the 32CA reaction between 2,5dimethyl-2H-[1,2,3]diazaphosphole $\mathbf{1}$ and acetonitrile $\mathrm{N}$-oxide $\mathbf{2}$ are studied; and finally, (iv) in the fourth part, the formation of the new single bonds along the most favourable reaction path associated with the formation of 3,5,7-trimethyl-5,7a-dihydro-[1,2,3]diazaphospholo[3,4-d][1,2,4]oxaphosphole 3 is characterized through the topological study of the ELF.

\subsection{Analysis of the CDFT indices of the reagents}

The 32CA reaction between 2,5-dimethyl-2H-[1,2,3]diazaphosphole $\mathbf{1}$ and acetonitrile N-oxide $\mathbf{2}$ was investigated analyzing the CDFT reactivity indices of the reagents. The electronic chemical potential $\mu$, the chemical hardness $\eta$, the electrophilicity $\omega$, and the nucleophilicity $N$, are given in Table 1 .

Referring to the values obtained for the electronic chemical potential, we observe that the value of electronic chemical potential of acetonitrile $\mathrm{N}$-oxide $\mathbf{2}$, $-2.89 \mathrm{eV}$, is higher than that of 2,5-dimethyl-2H$[1,2,3]$ diazaphosphole $\mathbf{1},-3.65 \mathrm{eV}$, indicating that along a polar reaction, the electron density will flux

Table 1. B3LYP/6-31G(d,p) the chemical hardness $\eta$, electronic chemical potential $\mu$, the electrophilicity $\omega$, and the nucleophilicity $N$, in $\mathrm{eV}$, of 2,5-dimethyl-2H-[1,2,3]diazaphosphole $\mathbf{1}$ and acetonitrile N-oxide 2.

\begin{tabular}{ccccc}
\hline System & $\eta$ & $\mu$ & $\omega$ & $\mathrm{N}$ \\
\hline $\mathbf{1}$ & 5.38 & -3.65 & 1.24 & 2.76 \\
$\mathbf{2}$ & 7.66 & -2.89 & 0.54 & 2.39 \\
\hline
\end{tabular}

from the acetonitrile $\mathrm{N}$-oxide $\mathbf{2}$ framework towards the 2,5-dimethyl-2H-[1,2,3]diazaphosphole one.

The electrophilicity $\omega$ and nucleophilicity $\mathrm{N}$ index of the 2,5-dimethyl-2H-[1,2,3]diazaphosphole $\mathbf{1}$ are 1.24 and $2.76 \mathrm{eV}$, respectively, and is classified as a strong electrophile and a moderate nucleophile within the electrophilicity and nucleophilicity scales. The electrophilicity $\omega$ and nucleophilicity $N$ indices of acetonitrile $\mathrm{N}$-oxide 2 are 0.54 and $2.39 \mathrm{eV}$, respectively, being classified marginal electrophile as a poor nucleophile. Consequently, it is expected that acetonitrile $\mathrm{N}$-oxide 2 participates as a poor nucleophile towards the electrophilic 2,5-dimethyl-2H-[1,2,3]diazaphosphole 1 in this zw-type 32CA reaction. However, analysis of the GEDT at the most favorable TS1 indicates that the GEDT fluxes from 1 towards 2 , the former acting as a nucleophile and the nitrile oxide as an electrophile (see later).

Because of the non-symmetric reagents, the most favorable regioisomeric reactive channel is the one that relates to the initial two-centre interaction between the most electrophilic center of the electrophile and the most nucleophilic center of the nucleophile. The nucleophilic $P_{k}^{-}$and electrophilic $P_{k}^{+}$ Parr functions, ${ }^{28}$ derived from the changes of spin electron-density reached via the GEDT process from the nucleophile to the electrophile, were recently proposed as a powerful tool to study the local reactivity in polar and ionic processes. Accordingly, the nucleophilic $P_{k}^{-}$and electrophilic $P_{k}^{+}$Parr functions of the 2,5-dimethyl-2H-[1,2,3]diazaphosphole $\mathbf{1}$ and acetonitrile $\mathrm{N}$-oxide $\mathbf{2}$, respectively, were analyzed in order to define the most electrophilic and nucleophilic centers of the species elaborate in this cycloaddition reaction and, thus, to explicate the regioselectivity experimentally detected (Figure 1).

After the nucleophilic $P_{k}^{-}$Parr functions analysis at the reactive sites of 2,5-dimethyl-2H-[1,2,3]diazaphosphole $\mathbf{1}$ it can be concluded that the $\mathrm{P}$ atom is the most nucleophilic center of this species with a $P_{k}^{-}$ value of $0.41(\mathrm{P})$. Otherwise, the electrophilic $P_{k}^{+}$Parr functions analysis at the reactive sites of acetonitrile $\mathrm{N}$-oxide 2 reveals that the carbon $\mathrm{C}^{\prime}$ carbon, $P_{k}^{+}=$ 0.30 , is significantly more nucleophilically activated than oxygen atom, $P_{k}^{+}=0.09$. Therefore, it is predictable that the most favorable nucleophile-electrophile interaction along the nucleophilic attack of 2,5-dimethyl-2H-[1,2,3]diazaphosphole $\mathbf{1}$ to acetonitrile $\mathrm{N}$-oxide $\mathbf{2}$ in a polar process will take place between the most electrophilic center of 2,5-dimethyl$2 \mathrm{H}-[1,2,3]$ diazaphosphole $\mathbf{1} \mathrm{P}$ phosphor atom and the most nucleophilic center of acetonitrile $\mathrm{N}$-oxide, the 

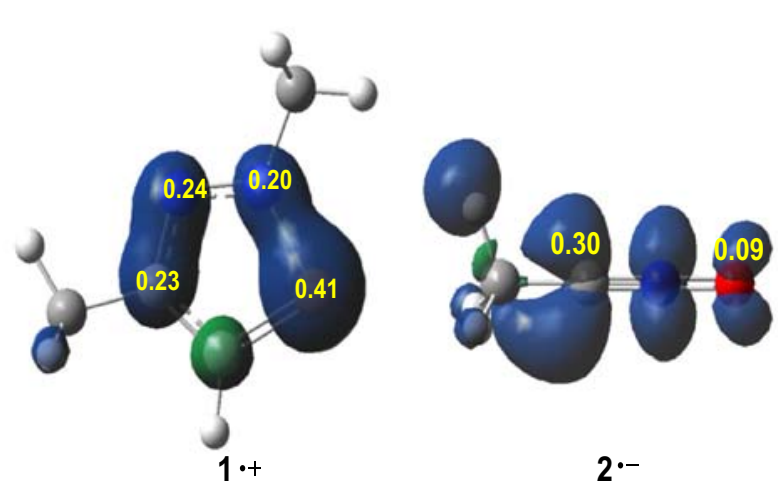

Figure 1. Three-dimensional (3D) representations of the Mulliken atomic spin densities (ASD) of radical cation $\mathbf{1}^{+}$, and radical anion $2^{-}$, together with the nucleophilic $P_{k}^{-}$and electrophilic $P_{k}^{+}$Parr functions, respectively, of the 2,4dimethyl-2H-phosphorus $\mathbf{1}$ and acetonitrile N-oxide 2.

$\mathrm{C}^{\prime}$ carbon atom. This prediction is in complete agreement with the experimental outcomes.

\subsection{ELF analysis of the electronic structure of acetonitrile $\mathrm{N}$-oxide}

Nitrile oxides are lineal TACs with a propargylic zwitterionic structure participating in zw-type 32CA reactions. ${ }^{9}$ Regardless of the electron-releasing or electron-withdrawing nature of the nitrile oxide substitution, these TACs usually exhibit a relatively low reactivity and yield low regioselectivity. Illustration of the ELF attractor positions and the proposed ELFbased Lewis structure of the acetonitrile $\mathrm{N}$-oxide $\mathbf{2}$ are illustrated in Figure 2.

The topological analysis of the ELF of acetonitrile N-oxide 2 shows the presence of two disynaptic basins, $\mathrm{V}(\mathrm{C} 1, \mathrm{~N} 2)$ and $\mathrm{V}(\mathrm{N} 2, \mathrm{O} 1)$, integrating 2.03 and 1.58 e, respectively, associated with the $\mathrm{C} 1-\mathrm{N} 2$ and $\mathrm{N} 2-\mathrm{O} 3$ bonding regions, as well as three $\mathrm{V}(\mathrm{C} 1, \mathrm{~N} 2)$ disynaptic basins, integrating a total of $6.23 \mathrm{e}$, associated to the $\mathrm{C} 1-\mathrm{N} 2$ triple bonding regions, and three $\mathrm{V}(\mathrm{O} 1)$ monosynaptic basins, integrating a total of 5.69 $\mathrm{e}$, related to the $\mathrm{O} 1$ non-bonding region (Figure 1).

NPA of acetonitrile $\mathrm{N}$-oxide 2 reveals that while the $\mathrm{O} 3$ oxygen gathers a relatively high negative charge, $-0.41 \mathrm{e}$, the $\mathrm{N} 2$ nitrogen is positively charged by 0.15 $\mathrm{e}$; for its part, the $\mathrm{C} 3$ carbon is also positively charged by 0.17 e. These values indicate that acetonitrile $\mathrm{N}$-oxide 2 presents a charge separation that makes them some dipolar character.

The proposed ELF-based Lewis structure of acetonitrile $\mathrm{N}$-oxide $\mathbf{2}$ indicates that it has a propargylic lineal structure associated with a $z w$-type TAC participation in zw-type 32CA reaction.

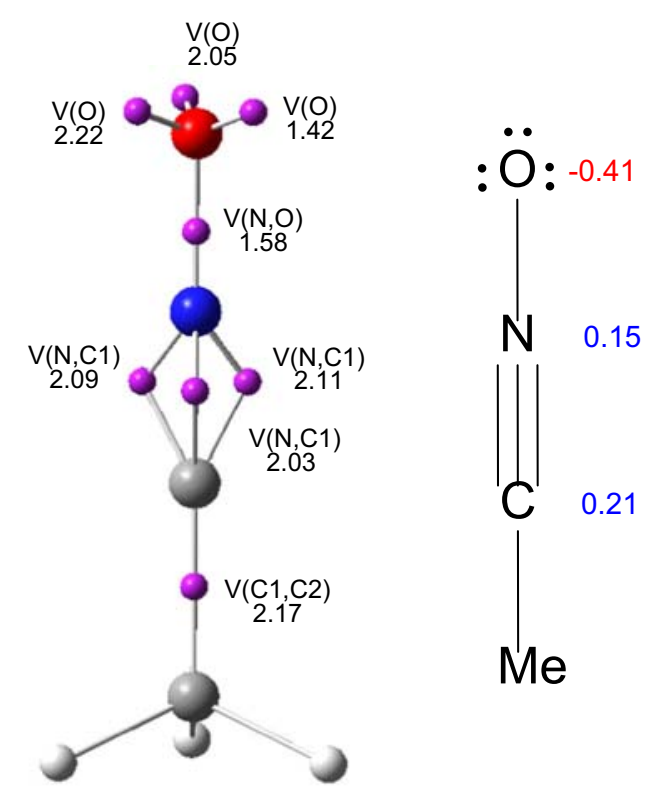

Figure 2. B3LYP/6-31G(d,p) ELF basin attractor positions, together with the valence basin populations, and the proposed ELF-based Lewis structure, together with the natural atomic charges of acetonitrile $\mathrm{N}$-oxide 2. Negative charges are colored in red, while positive charges are colored in blue. ELF valence basin populations and natural atomic charges are given in an average number of electrons, e.

\subsection{Study of the competitive reaction paths} associated with the 32CA reaction between 2,5dimethyl-2H-[1,2,3]diazaphosphole 1 and acetonitrile $\mathrm{N}$-oxide 2

2,5-dimethyl-2H-[1,2,3]diazaphosphole $\mathbf{1}$ has two reactive sites: one $\mathrm{P}-\mathrm{C}$ double bond and one $\mathrm{C}-\mathrm{N}$ double bond. Due to the non-symmetry of 2,5-dimethyl-2H-[1,2,3]diazaphosphole $\mathbf{1}$ and acetonitrile $\mathrm{N}$-oxide 2, up to four competitive reaction paths are feasible. They are related to the different chemo- and regioisomeric approach modes of acetonitrile $\mathrm{N}$-oxide 2 to 2,5-dimethyl-2H-[1,2,3]diazaphosphole 1. Formation of 3,5,7-Trimethyl-5,7a-dihydro-[1,2,3]diazaphospholo[3,4-d][1,2,4] oxaphosphole 3 as the single reaction product indicates that this 32CA reaction has to present total region and chemoselectivity.

So as to explicate the regio- and chemoselectivity experimentally observed, two approach modes associated to the chemoselective attack on the P-C2 and N1-C3 double bonds of 2,5-dimethyl-2H-[1,2,3]diazaphosphole $\mathbf{1}$ along with the two regioisomeric approach modes of acetonitrile $\mathrm{N}$-oxide $\mathbf{2}$, were considered. Thus, four TSs, TS1 to TS4, and the corresponding cycloadducts were located and characterized (Scheme 3), demonstrating that the aforementioned reaction paths proceed through a one-step mechanism. 


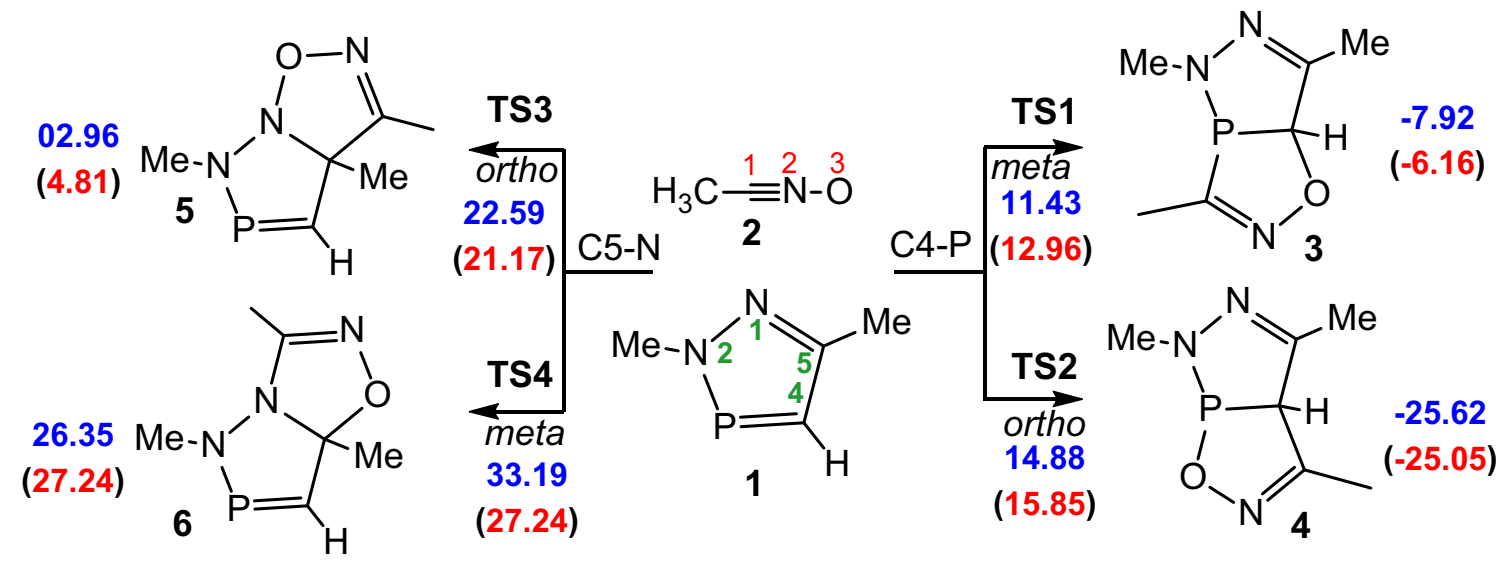

Scheme 3. The four considered regio- and chemo isomeric reaction paths associated with the 32CA reaction between 2,5dimethyl-2H-[1,2,3]diazaphosphole 1 and acetonitrile $\mathrm{N}$-oxide 2. B3LYP/6-31G(d,p) relative energies, in the gas phase and in $\mathrm{THF}$ (the latter in parentheses), are given in $\mathrm{kcal} \cdot \mathrm{mol}^{-1}$ relative to the separated reagents.

Relative energies, in the gas phase and in THF, are given in Scheme 3, while total electronic energies are given in Table S1 (Supplementary Information).

The activation energies related with the four selected reaction paths range from 11.43 (TS3) to 33.19 (TS5) $\mathrm{kcal} \cdot \mathrm{mol}^{-1}$; formation of the corresponding cycloadducts being two exothermic by between 7.92 (3) and $25.62(4) \mathrm{kcal} \cdot \mathrm{mol}^{-1}$, and two endothermic by 02.96 (5) and 26.35 (6). Several engaging conclusions can be drawn from these energy results: (i) the most favourable reaction path is that related to the formation of 3 via TS1, $11.43 \mathrm{kcal} \cdot \mathrm{mol}^{-1}$. This reaction path corresponds to the approach of acetonitrile $\mathrm{N}$-oxide 2 towards the $\mathrm{P}_{-} \mathrm{Cl}^{\prime}$ double bond of 2,4-dimethyl-2Hphosphorus 1; (ii) this 32CA reaction presents a total chemoselectivity as TS3 is $11.16 \mathrm{kcal} \cdot \mathrm{mol}^{-1}$ higher in energy than TS1; (iii) this 32CA reaction presents a total regioselectivity as TS2 is $3.45 \mathrm{kcal} \cdot \mathrm{mol}^{-1}$ higher in energy than TS1; (vi) formation of cycloadduct $\mathbf{4}$ is more exothermic than the formation of cycloadduct $\mathbf{3}$; -25.26 and $-7.92 \mathrm{kcal} \cdot \mathrm{mol}^{-1}$, respectively. Therefore, it is expected that product 3 results from a kinetic control of the reaction.

Inclusion of the solvent effects of THF produces negligible changes in activation and reaction energies of the products $\mathbf{3}, \mathbf{4}$ and $\mathbf{5}$ between only 0.97 and 1.53 $\mathrm{kcal} \cdot \mathrm{mol}^{-1}$, but in the fourth activation energy of product 6 a very large load of $5.95 \mathrm{kcal} \mathrm{mol}^{-1}$. Whilst in THF the activation energies slightly increase and the exothermic character of the reaction paths a little diminish, as a result of better solvation of the reagents than that of the TSs and cycloadducts. Consequently, in THF, TS1 and TS2 present alike activations energies. The outcomes found in the gas phase are not modified in THF.
The enthalpy profiles of the reaction paths associated with the 32CA reaction between 2,5-dimethyl2H-[1,2,3]diazaphosphole $\mathbf{1}$ and acetonitrile $\mathrm{N}$-oxide $\mathbf{2}$ are illustrated in Figure 2, while the complete thermodynamic data are given in Table S2 (Supplementary Information). Again, the addition of thermal corrections to the electronic energies in THF does not considerably change the relative enthalpies; while the activation enthalpies slightly increase by ca. 1.0 $\mathrm{kcal} \cdot \mathrm{mol}^{-1}$, the reaction enthalpies slightly decrease by ca. $3.0 \mathrm{kcal} \cdot \mathrm{mol}^{-1}$. Though, the inclusion of entropies to enthalpies strongly increases the activation of Gibbs free energies by between 11-12 $\mathrm{kcal} \cdot \mathrm{mol}^{-1}$, due to the unfavourable entropies associated with these biomolecular processes. The activation Gibbs free energies associated with the formation of $\mathbf{3}$ and 4 reach 25.2 and $28.27 \mathrm{kcal} \cdot \mathrm{mol}^{-1}$, respectively. The activation Gibbs free energies also account for the total chemo- and regioselectivity.

The geometries of the TSs associated with the four reaction paths are exposed in Figure 3. The comparative scrutiny of the geometrical parameters of the TSs found in the gas phase and those found in THF demonstrate that the implicit consideration of the solvent in the geometry optimisations does not create significant changes. Some attractive conclusions can be drawn from the geometrical parameters of the TSs illustrate in Figure 3: (i) the lengths of the two forming bonds at the TSs associated with the reaction paths involving the participation of the $\mathrm{P}-\mathrm{C}$ double bond of 2,5-dimethyl-2H-[1,2,3]diazaphosphole $\mathbf{1}$ show that they are associated with asynchronous processes in which the formation of the P-C1 single bond involving the $\mathrm{P}-\mathrm{C}$ double bond phosphor atom is more advanced than the double bond $\mathrm{N}-\mathrm{C}$; (ii) along the two 


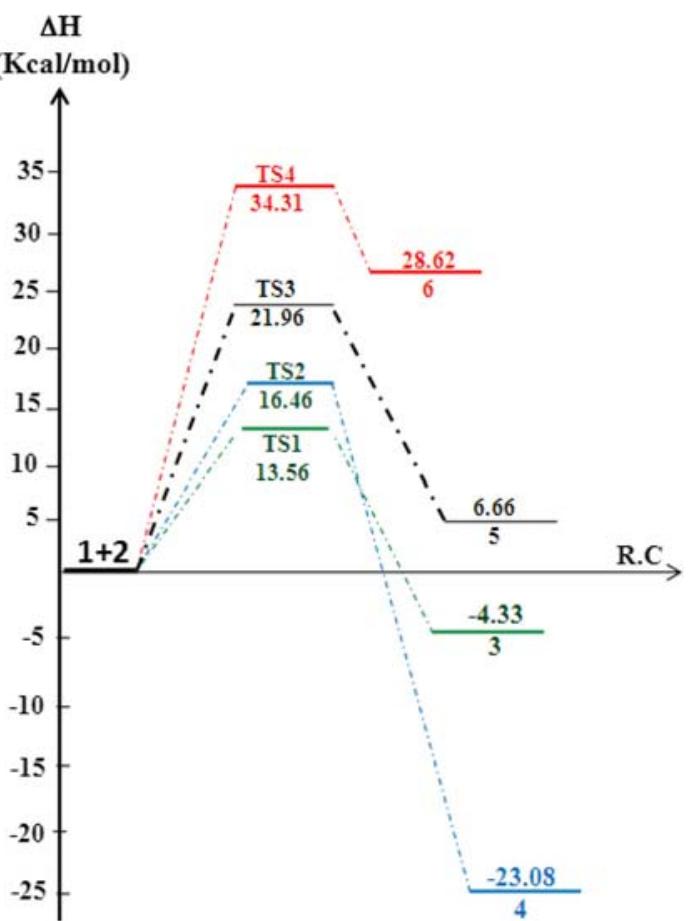

Figure 3. Enthalpy profiles, in $\mathrm{kcal} \cdot \mathrm{mol}^{-1}$, for the studied reaction paths of the 32CA 2,5-dimethyl-2H-[1,2,3]diazaphosphole $\mathbf{1}$ and acetonitrile $\mathrm{N}$-oxide 2 , in $\mathrm{THF}$ at $25^{\circ} \mathrm{C}$ $\left(\mathrm{kcal} \cdot \mathrm{mol}^{-1}\right)$.

chemoisomeric reaction paths involving the participation of the P-C double bond of 2,5-dimethyl-2H[1,2,3]diazaphosphole $\mathbf{1}$, the more favourable TS1 is more advanced and more asynchronous than TS3; and finally, (iii) inclusion of the solvent effects makes the TSs slightly more earlier and more asynchronous.

Finally, the polar character of these 32CA reactions was evaluated computing the values of the GEDT at the corresponding TSs. The values of the GEDT, which flux from diazaphosphole framework to the nitrile oxide one are $0.18 \mathrm{e}$ at TS1, $0.01 \mathrm{e}$ at TS2, 0.05 e at TS3, 0.17 e at TS4 and 0.11 e at TS4. Thus, most favourable TS1 is the most polar one. The corresponding EDT value indicates that this zw-type 32CA has polar character (Figure 4).

\subsection{ELF topological analysis of the $\mathrm{P}-\mathrm{C}$ and $\mathrm{C}-\mathrm{O}$} bond formation along with the $32 \mathrm{CA}$ reaction of between 2,5-dimethyl-2H-[1,2,3]diazaphosphole 1 and acetonitrile $\mathrm{N}$-oxide

In order to characterise the $\mathrm{P}-\mathrm{C}$ and $\mathrm{C}-\mathrm{O}$ bond formation along with the 32CA reaction between 2,5dimethyl-2H-[1,2,3]diazaphosphole $\mathbf{1}$ and acetonitrile $\mathrm{N}$-oxide 2, a topological analysis of the ELF along the IRC associated with the most favourable ortho reaction path was performed. The IRC structures directly
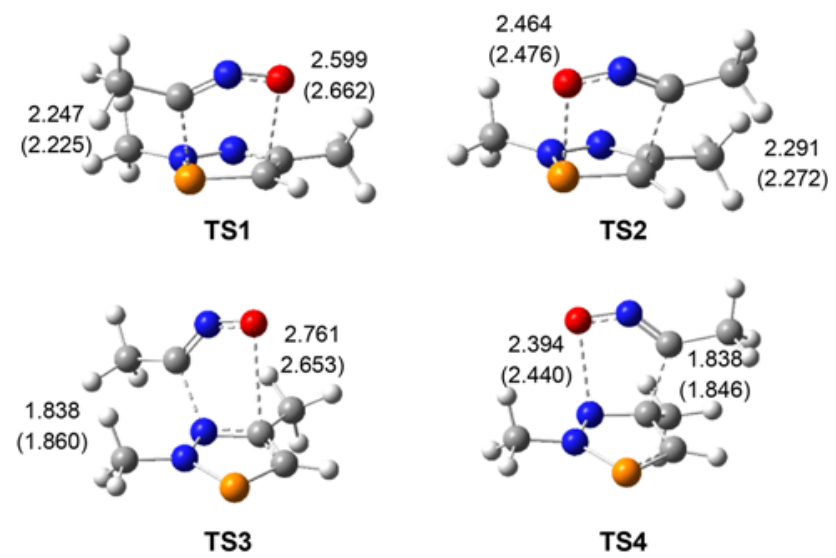

Figure 4. B3LYP/6-31G(d,p) optimised geometries of the TSs involved in the four considered chemo-and regioisomeric reaction paths associated with the $32 \mathrm{CA}$ reaction between 2,5-dimethyl-2H-[1,2,3]diazaphosphole $\mathbf{1}$ and acetonitrile N-oxide 2. Distances are given in angstroms; $\AA$. Distances in THF are given in parentheses.

involved in the formation of the new $\mathrm{P}-\mathrm{C}$ and $\mathrm{C}-\mathrm{O}$ single bonds were selected by performing the topological analysis of the ELF for all the structures of the IRC having $2.6 \AA>\mathrm{d}(\mathrm{P}-\mathrm{C})>1.9 \AA$ and $2.9>\mathrm{d}(\mathrm{O}-\mathrm{C})$ $>$ 1.6. The complete ELF analysis is given in the Supplementary Information, while the attractor positions of the ELF basins are given in Figure S1 (Supplementary Information).

Some appealing conclusions can be drawn from this ELF topological study: (i) the relatively high activation energy of the 32CA reaction between 2,5-dimethyl-2H-[1,2,3]diazaphosphole $\mathbf{1}$ and acetonitrile N-oxide 2, $11.43 \mathrm{kcal} \cdot \mathrm{mol}^{-1}$ for TS1, can mainly be related to the rupture of the $\mathrm{C} \equiv \mathrm{N}$ triple bond of the nitrile oxide framework, which conduct the formation of non-bonding electron density at the $\mathrm{N}$ nitrogen and the $\mathrm{C} 1$ carbon; (ii) formation of the first P-C single bond takes place at a P-C distance of ca. $1.95 \AA$ with an initial population of 1.22 e by sharing part of the non-bonding electron density of the $\mathrm{P}$ center and that of the C pseudo radical center (see P5 in Figure 5); (iii) formation of the second $\mathrm{O}-\mathrm{C}$ single bond takes place at a C-O distance of ca. $1.5 \AA$, with an initial population of $0.73 \mathrm{e}$, by donating some $\mathrm{O} 3$ oxygen non-bonding electron density to the $\mathrm{C} 5$ carbon (see $\mathbf{P 6}$ Figure 5); and finally (iv) taking into account the IRC values of the structures at which formation of the two single bonds occurs, i.e., P5 and P6, the bond formation can be considered highly asynchronous. Note that the formation of the second $\mathrm{O}-\mathrm{C}$ single bond begins when the formation of the first $\mathrm{P}-\mathrm{C}$ single bond is completed by $100 \%$. This behaviour indicates that this 32CA reaction takes place through a non-concerted two-stage one-step mechanism. ${ }^{32}$ 


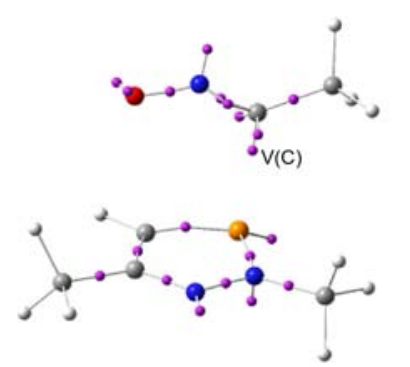

TS1

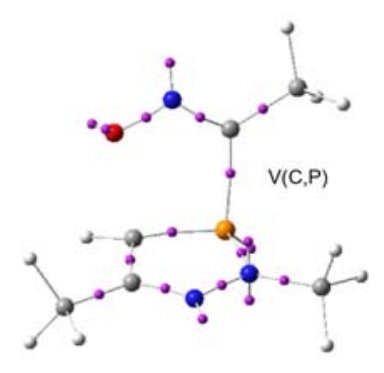

P5

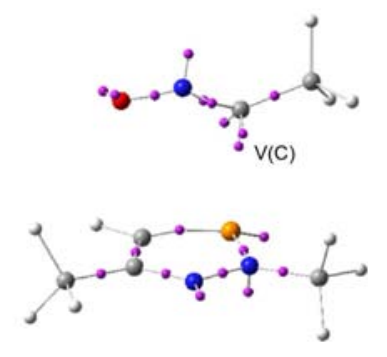

P3

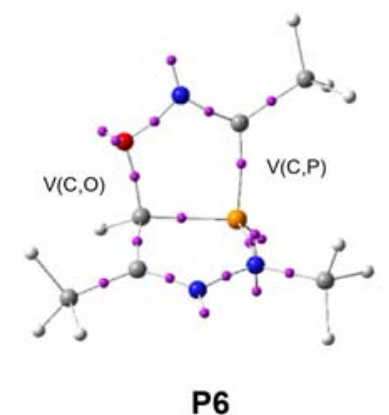

Figure 5. ELF attractor positions for the structures involved in the formation of the $\mathrm{P}-\mathrm{C}$ and $\mathrm{C}-\mathrm{O}$ single bonds along the most favourable TS1 reaction path associated with the 32CA reaction between 2,5-dimethyl-2H-[1,2,3]diazaphosphole $\mathbf{1}$ and acetonitrile $\mathrm{N}$-oxide 2.

\section{Conclusions}

The 32CA reaction of 2,5-dimethyl-2H-[1,2,3]diazaphosphole 1 with acetonitrile $\mathrm{N}$-oxide 2 yielding 3,5,7Trimethyl-5,7a-dihydro-[1,2,3]diazaphospholo[3,4d] $[1,2,4]$ oxaphosphole $\mathbf{3}$, has been studied within MEDT through DFT calculations at the B3LYP/631(d,p) computational level. Due to the non-symmetry of $\mathbf{1}$ and acetonitrile $\mathrm{N}$-oxide $\mathbf{2}$, up to four competitive reaction paths are feasible. The chemo- and regioisomeric reaction paths involving the two double bonds $\mathrm{P}-\mathrm{C}$ and $\mathrm{C}-\mathrm{N}$ of 2,5-dimethyl-2H-[1,2,3]diazaphosphole 1 have been studied.

Analysis of the activation energies indicates that this 32CA reaction is completely chemo- and regioselective, under kinetic control. Analysis of the ELF of the selected points of the IRC associates with the formation of the new $\mathrm{P}-\mathrm{C}$ and $\mathrm{C}-\mathrm{O}$ single bonds along with the ortho reaction path accounts of the zw-type reactivity of acetonitrile N-oxide 2. ELF analysis indicates that the reaction begins with the formation of the $\mathrm{P}-\mathrm{C}$ single bond involving the phosphor double bond and the $\mathrm{C} 1$ carbon of acetonitrile $\mathrm{N}$-oxide 2 , after passing the TS. Formation of the second $\mathrm{C}-\mathrm{O}$ single bond takes place at the end of the reaction, thus characterizing the mechanism of this 32CA reaction by a non-concerted one-step two-stage.

\section{Supplementary Information (SI)}

ELF topological analysis of the $\mathrm{P}-\mathrm{C}$ and $\mathrm{C}-\mathrm{O}$ bond formation along with the 32CA reaction between 2,5dimethyl-2H-[1,2,3]diazaphosphole $\mathbf{1}$ and acetonitrile N-oxide 2. Tables include B3LYP/6-31g(d,p) total and relative electronic energies, in the gas phase and in THF, and B3LYP/6-31G(d,p) thermodynamic data, computed at $25^{\circ} \mathrm{C}$ and $1 \mathrm{~atm}$ in THF, for the stationary points involved in the 32CA reaction of 2,4-dimethyl-2H-phosphorus 1 with acetonitrile N-oxide 2. Supplementary information is available at www.ias.ac.in/chemsci.

\section{References}

1. Moriya $\mathrm{O}$, Takenaka $\mathrm{P} \mathrm{H}$, lyoda $\mathrm{M}$, Urataa $\mathrm{Y}$ and Endob T 1994 Generation of nitrite oxides via O-tributylstannylaldoximes; application to the synthesis of isoxazolines and isoxazoles J. Chem. Soc. Perkin. Trans. I 0413

2. Li X, Wang X, Wang Z, Yan X and Xu X 2019 TBHPinduced iodocyclization with I2: atom economic synthesis of iodinated isoxazolines in water under mild conditions ACS Sustain. Chem. Eng. 71875

3. Woods D J, Vaillancourt V A, Wendt J A and Meeus P F 2011 Discovery and development of veterinary antiparasitic drugs: past, present and future Future Med. Chem. 3887

4. Zaki M, Oukhrib A, Akssira M and Berteina-Raboin S 2017 Synthesis of novel spiro-isoxazoline and spiroisoxazolidine derivatives of tomentosin $R S C A d v .7$ 6523

5. Zheng Y, Tice C M and Singh S B 2014 The use of spirocyclic scaffolds in drug discovery Bioorg. Med. Chem. Lett. 243673

6. Yeung Lam KoY Y C, Tonnard F, Carrié R, De Sarlo F and Brandi A 1983 Cycloaddition d'oxydes de nitrile a des diazaphospholes et des composés apparentés. Réactivité comparée des doubles liaisons $\mathrm{N}=\mathrm{C}, \mathrm{P}=\mathrm{C}$ et As=C. Tetrahedron 391507

7. Domingo L R 2016 Molecular electron density theory: a modern view of reactivity in organic chemistry Molecules 211319

8. Ríos-Gutiérrez M and Domingo L R 2019 Unravelling the mysteries of the [3+2] cycloaddition reactions Eur. J. Org. Chem. 267

9. Domingo L R, Aurell M J and Pérez P 2014 A DFT analysis of the participation of zwitterionic TACs in polar [3+2] cycloaddition reactions Tetrahedron $\mathbf{7 0}$ 4519

10. Ndassa I M, Adjieufack A I, Ketcha J M, Berski S, Ríos-Gutiérrez M and Domingo L R 2017 Understanding the reactivity and regioselectivity of $[3+2]$ cycloaddition reactions between substituted nitrile oxides and methylacrylate. A molecular electron density theory study Int. J. Quantum Chem. 11725451

11. Lee C, Yang W and Parr R G 1988 Development of the Colle-Salvetti correlation-energy formula into a functional of the electron density Phys. Rev. B 37785 
12. Becke A D 1993 Density-functional thermochemistry. III. The role of exact exchange J. Chem. Phys. 98 5648

13. Hehre W J, Radom L, Schleyer P v R and Pople J A 1986 Ab initio Molecular Orbital Theory (New York: Wiley)

14. Schlegel H B 1982 Optimization of equilibrium geometries and transition structures J. Comput. Chem. 2214

15. Schlegel H B 1994 In Modern Electronic Structure Theory D R Yarkony (Ed.) (Singapore: World Scientific Publishing)

16. K Fukui 1970 Formulation of the reaction coordinate $J$. Phys. Chem. 744161

17. González C and Schlegel H B 1990 Reaction path following in mass-weighted internal coordinates $J$. Phys. Chem. 945523

18. González C and Schlegel H B 1991 Improved algorithms for reaction path following: higher-order implicit algorithms J. Chem. Phys. 955853

19. Tomasi J and Persico M 1994 Molecular interactions in solution: an overview of methods based on continuous distributions of the solvent Chem. Rev. 942027

20. Cossi M, Barone V, Cammi R and Tomasi J 1996 Ab initio study of solvated molecules: a new implementation of the polarizable continuum model Chem. Phys. Lett. 255 327

21. Cances E, Mennucci B and Tomasi J 1997 Evaluation of solvent effects in isotropic and anisotropic dielectrics and in ionic solutions with a unified integral equation method: theoretical bases, computational implementation, and numerical applications J. Chem. Phys. 1073032
22. Barone V, Cossi M and Tomasi J 1998 Geometry optimization of molecular structures in solution by the polarizable continuum model J. Comput. Chem. 19404

23. Domingo L R 2014 A new $\mathrm{C}-\mathrm{C}$ bond formation model based on the quantum chemical topology of electron density RSC Adv. 432415

24. Reed A E, Weinstock R B and Weinhold F 1985 Natural population analysis J. Chem. Phys. 83735

25. Reed A E, Curtiss L A and Weinhold F 1988 Intermolecular interactions from a natural bond orbital, donor-acceptor viewpoint Chem. Rev. 88899

26. Parr R G, Szentpaly, L V and Liu S 1999 Electrophilicity Index J. Am. Chem. Soc. 1211922

27. Domingo L R, Chamorro E and Pérez P 2008 Understanding the reactivity of captodative ethylenes in polar cycloaddition reactions. A theoretical study J. Org. Chem. 734615

28. Domingo L R, Pérez P and Sáez J A 2013 Understanding the local reactivity in polar organic reactions through electrophilic and nucleophilic Parr functions RSC Adv. 31486

29. M J Frisch et al., Gaussian 09, Inc., Wallingford CT, 2009

30. Becke A D and Edgecombe K E 1990 A simple measure of electron localization in atomic and molecular systems J. Chem. Phys. 925397

31. Noury S, Krokidis X, Fuster F and Silvi B 1999 Computational tools for the electron localization function topological analysis Comput. Chem. 23597

32. Domingo L R, Saez J A, Zaragoza R J and Arno M 2008 Understanding the participation of quadricyclane as nucleophile in polar $[2$ sigma +2 sigma +2 pi] cycloadditions toward electrophilic pi molecules $J$. Org. Chem. 738791 